\title{
Multi-frequency investigation of the parsec- and kilo-parsec-scale radio structures in high-redshift quasar PKS 1402+044
}

\author{
J. Yang ${ }^{1,2}$, L.I. Gurvits ${ }^{3}$, A.P. Lobanov ${ }^{4}$, S. Frey ${ }^{5,6}$, and X.-Y. Hong ${ }^{1}$ \\ 1 Shanghai Astronomical Observatory, Chinese Academy of Sciences, 80 Nandan Road, 200030 Shanghai, PR China \\ e-mail: junyang@shao.ac.cn \\ 2 Graduate University of the Chinese Academy of Sciences, Beijing, PR China \\ 3 Joint Institute for VLBI in Europe, PO Box 2, 7990 AA Dwingeloo, The Netherlands \\ e-mail: lgurvits@jive.nl \\ 4 Max-Planck-Institut für Radioastronomie, Auf dem Hügel 69, 53121 Bonn, Germany \\ e-mail: alobanov@mpi fr-bonn.mpg.de \\ 5 FÖMI Satellite Geodetic Observatory, PO Box 585, 1592 Budapest, Hungary \\ e-mail: frey@sgo.fomi.hu \\ ${ }^{6}$ MTA Research Group for Physical Geodesy and Geodynamics, PO Box 91, 1521 Budapest, Hungary
}

Received 26 March 2008 / Accepted 16 June 2008

\section{ABSTRACT}

\begin{abstract}
Aims. We investigate the frequency-dependent radio properties of the jet of the luminous high-redshift $(z=3.2)$ radio quasar PKS 1402+044 (J1405+0415) by means of radio interferometric observations.

Methods. The observational data were obtained with the VLBI Space Observatory Programme (VSOP) at 1.6 and $5 \mathrm{GHz}$, supplemented by other multi-frequency observations with the Very Long Baseline Array (VLBA; 2.3, 8.4, and $15 \mathrm{GHz}$ ) and the Very Large Array (VLA; $1.4,5,15$, and $43 \mathrm{GHz}$ ). The observations span a period of 7 years.

Results. We find that the luminous high-redshift quasar PKS 1402+044 has a pronounced "core-jet" morphology from the parsec to the kilo-parsec scales. The jet shows a steeper spectral index and lower brightness temperature with increasing distance from the jet core. The variation of brightness temperature agrees well with the shock-in-jet model. Assuming that the jet is collimated by the ambient magnetic field, we estimate the mass of the central object as $\sim 10^{9} M_{\odot}$. The upper limit of the jet proper motion of PKS $1402+044$

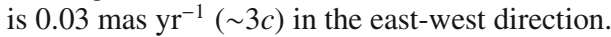

Key words. galaxies: individual: PKS 1402+044 - galaxies: active - galaxies: quasars: general - galaxies: jets - radio continuum: galaxies

\section{Introduction}

Very Long Baseline Interferometry (VLBI) studies of highredshift quasars at a given observing frequency $v_{\mathrm{obs}}$ can facilitate comparison of their structural properties with those of their lower-redshift counterparts at a higher frequency, $v_{\mathrm{obs}}=$ $v_{\mathrm{em}} /(1+z)$, where $v_{\mathrm{em}}$ is the emitted (rest-frame) frequency and $z$ the redshift of a distant quasar. High-redsfhit quasars provide indispensable input in all kinds of studies of the redshift-dependent properties of extragalactic objects, such as the apparent "angular size - redshift" (" $\theta-z$ ", e.g. Gurvits et al. 1999) and "proper motion - redshift" (" $\mu-z$ ", e.g. Kellermann et al. 1999) relations.

A statistical study of 151 quasars imaged with VLBI at $5 \mathrm{GHz}$ (Frey et al. 1997) demonstrated an overall trend of a decreasing jet-to-core flux density ratio with increasing redshift, which could be explained by the difference in spectral indices of cores and jets. Furthermore, a majority of radio QSOs at $z>4$ seemed to be even more compact than expected from the direct extrapolation of the properties of quasars at $z<4$ (Paragi et al. 1999).

A number of morphological studies of high-redshift objects have been made with the Japanese-led Space VLBI mission VSOP (VLBI Space Observatory Programme). Observations with the VSOP utilised an array consisting of a group of Earthbased radio telescopes and an $8 \mathrm{~m}$ space-borne antenna on board the satellite HALCA (Hirabayashi et al. 1998). The orbiting antenna with an apogee of $\sim 21000 \mathrm{~km}$ and perigee of $\sim 560 \mathrm{~km}$ provided milli-arcsecond (mas) and sub-mas resolution at the frequencies of 1.6 and $5 \mathrm{GHz}$. VSOP observations at $1.6 \mathrm{GHz}$ provided roughly the same angular resolution as Earth-based VLBI observations at $5 \mathrm{GHz}$. Thus, dual-frequency VSOP observations made it possible to map the distribution of spectral index across the source structure (e.g. Lobanov et al. 2006) and to study frequency-dependent structural properties.

PKS $1402+044(\mathrm{~J} 1405+0415)$ is a flat-spectrum radio source from the Parkes $2.7 \mathrm{GHz}$ Survey. In optics, it is a 19.6-magnitude ( $g$ filter) stellar object at a redshift of $z=3.215$. It is a weak $\mathrm{X}$-ray source with count rates of $(5.6 \pm 1.2) \times 10^{-3} \mathrm{ct} \mathrm{s}^{-1}$, over the band $0.2-4 \mathrm{keV}$ in the Einstein IPC survey database (Thompson et al. 1998) and $(1.3 \pm 0.2) \times 10^{-2} \mathrm{ct} \mathrm{s}^{-1}$ over the band $0.1-2.4 \mathrm{keV}$ in the ROSAT observation (Siebert et al. 1998). The MultiElement Radio Linked Interferometer Network (MERLIN) observations of PKS $1402+044$ made at $1.6 \mathrm{GHz}$ indicates that there is a secondary component at a separation of $0 .{ }^{\prime} 8$ at a position angle of $-123^{\circ}$ and a faint extended emission at a distance of $3^{\prime \prime} .3$ at a position angle of $-106^{\circ}$. VLBI observations at $5 \mathrm{GHz}$ (Gurvits et al. 1992) found that the main component consists of a compact core and a resolved jet extending up to $\sim 18$ mas to the west.

The quasar PKS 1402+044 represents a relatively rare case of a strong radio source at $z>3$ and therefore a potentially rewarding target for structural studies within a broad range of 
angular scales. VSOP observations with their record-high angular resolution at 1.6 and $5 \mathrm{GHz}$ facilitate direct comparison of structural properties of PKS $1402+044$ with its more abundant strong radio quasars at lower redshifts at the same emitting frequency.

In this paper, we present VSOP images at 1.6 and $5 \mathrm{GHz}$, a 15-GHz Very Long Baseline Array (VLBA) image, and Very Large Array (VLA) images at $1.4,5,15$, and $43 \mathrm{GHz}$ of the quasar PKS 1402+044; discuss its spectral properties and brightness temperature variation along the jet; and determine some physical parameters of the core and the jet. Throughout the paper, we define the spectral index $\alpha$ as $S_{v} \propto v^{\alpha}$ and adopt the $\Lambda$ CDM cosmological model (Riess et al. 2004) with $H_{0}=$ $75 \mathrm{~km} \mathrm{~s}^{-1} \mathrm{Mpc}^{-1}, \Omega_{\mathrm{m}}=0.3$, and $\Omega_{\Lambda}=0.7$. In the latter model, the linear scale factor for PKS $1402+044$ is $\sim 7 \mathrm{pc} \mathrm{mas}^{-1}$.

\section{Observations and data reduction}

\subsection{VSOP experiment}

Using the space-borne radio telescope HALCA and the VLBA, we observed the quasar PKS 1402+044 in left hand circular polarization at $5 \mathrm{GHz}$ on 20 January 2001 and at $1.6 \mathrm{GHz}$ on 21 January 2001. The observations lasted for $8 \mathrm{~h}$ at $1.6 \mathrm{GHz}$ and $7 \mathrm{~h}$ at $5 \mathrm{GHz}$. The data were recorded using the VLBA tape system with a $32 \mathrm{MHz}$ bandwidth consisting of 2 intermediate frequency (IF) bands and 2-bit sampling, corresponding to the data rate of $128 \mathrm{Mbps}$. Four tracking stations (Goldstone, Robledo, Tidbinbilla, Green Bank) were used to receive the HALCA downlink data. The HALCA data were recorded for $\sim 5 \mathrm{~h}$ at $1.6 \mathrm{GHz}$ and $4.2 \mathrm{~h}$ at $5 \mathrm{GHz}$. The data were correlated at the VLBA correlator in Socorro with 128 spectral channels and an integration time of $4.2 \mathrm{~s}$ for the ground-ground baselines, and $2.1 \mathrm{~s}$ at $1.6 \mathrm{GHz}, 1.0 \mathrm{~s}$ at $5 \mathrm{GHz}$ for the space-ground baselines. In the $1.6 \mathrm{GHz}$ observation, the Tidbinbilla station lost $55 \mathrm{~min}$ of space data and Green Bank lost all the space data (34 min) due to a problem with recording. At $5 \mathrm{GHz}$, the IF 1 data were lost for 40 min due to a technical malfunction at the Tidbinbilla station. Except for these problems, fringes were successfully detected on all the space-ground baselines at all times.

A priori calibration was applied for both datasets using the Astronomical Image Processing System (AIPS; Cotton 1995). After correcting the amplitudes in cross-correlation spectrum using measurements of auto-correlation spectrum and dispersive delay due to the ionosphere from the maps of total electron content, we applied a priori amplitude calibration from the antenna gain and system temperature measurements at the Earthbased telescopes. We used the respective nominal values ${ }^{1}$ for HALCA. After inspecting the IF bandpass, the side channels $(1-5,105-128)$ in each IF were deleted because of the lower amplitude $(<80 \%)$ than in the centre channels. This reduced the useful observing bandwidth to $22.75 \mathrm{MHz}$. Some channels affected by radio frequency interference were flagged, too. We corrected the residual delays and rates using a two-step fringefitting. We first fringe-fitted the ground-array data with a solution interval of $2 \mathrm{~min}$. Then we applied the solutions to the data, fixed the calibration for ground antennas and determined the calibration solutions of the space antenna using fringe-fitting with a 4 min interval. After that, we combined all fringe solutions, applied them to the data, averaged all the channels in each IF, and split the multi-source data into single-source data sets. Finally, the data were exported into Difmap (Shepherd et al. 1994) and

${ }^{1}$ http://www.vsop.isas. jaxa.jp/obs/HALCAcal.html

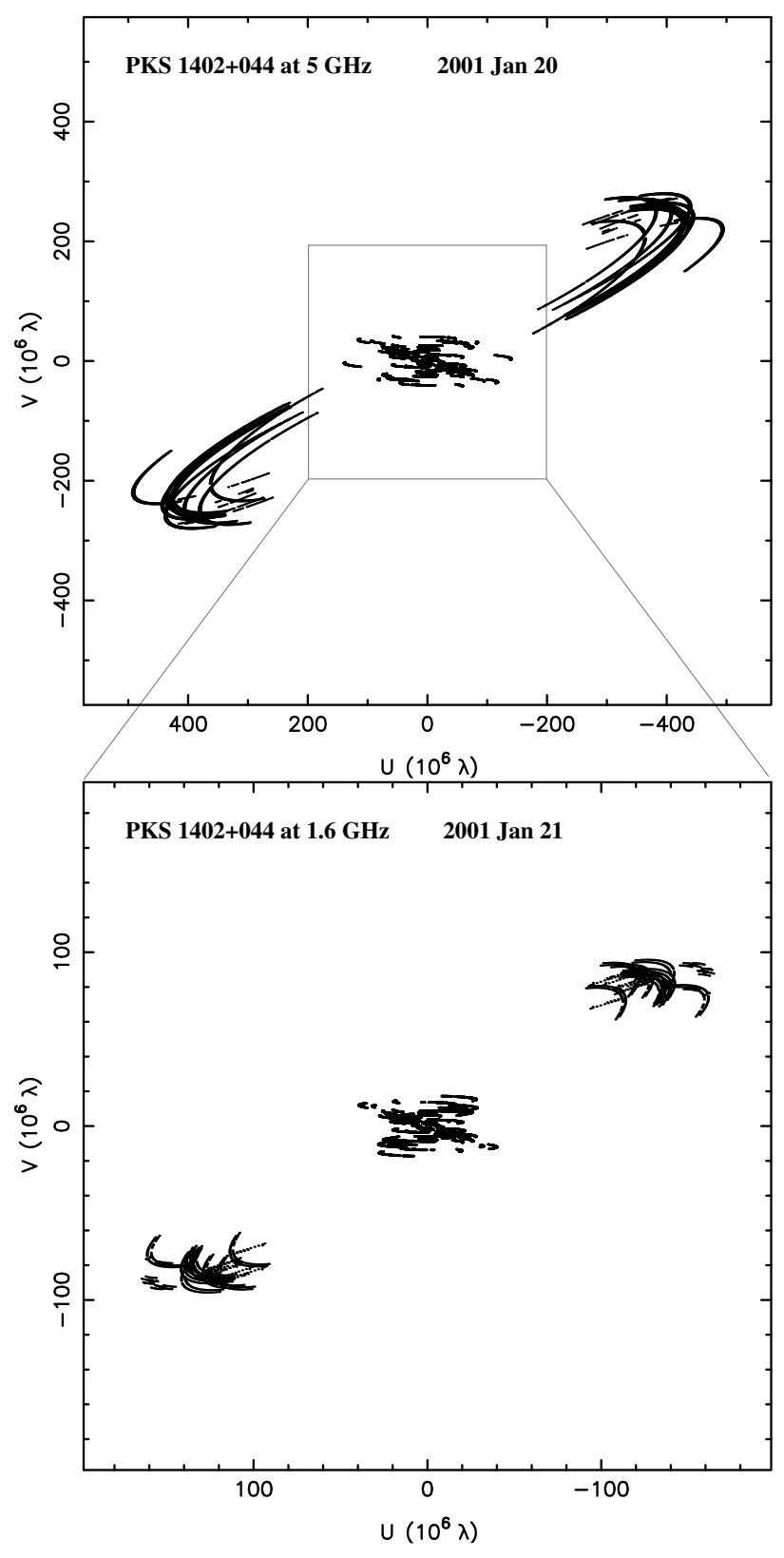

Fig. 1. The effective $(u, v)$ coverage of the VSOP observations of PKS $1402+044$ at $1.6 \mathrm{GHz}$ (bottom) and $5 \mathrm{GHz}$ (top). At each frequency, the inner tracks correspond to the ground-ground baselines, and the outer tracks denote the space-ground baselines. The rectangle in the $5-\mathrm{GHz}$ $(u, v)$ coverage shows the size of the $1.6 \mathrm{GHz}(u, v)$ coverage. The spaceground baselines provide $u v$-ranges roughly 2.5-3 times longer than ground-ground baselines.

averaged further over $60 \mathrm{~s}$ time intervals. The hybrid imaging and self-calibration were done in Difmap. The resulting effective $(u, v)$ coverages of the VSOP observations are shown in Fig. 1. The correlated flux densities as a function of the projected baseline length are displayed in Fig. 2, top and middle.

\subsection{VLBA and VLA data}

The $15 \mathrm{GHz}$ VLBA data presented in this paper are from the VLBA-VSOP support survey by Gurvits et al. (in preparation). The observations were conducted on 5 December 1998 with left-hand circular polarisation, $64 \mathrm{MHz}$ bandwidth and $\sim 50 \mathrm{~min}$ 
Table 1. VLA observations summary.

\begin{tabular}{ccccccc}
\hline \hline $\begin{array}{c}v_{\text {obs }} \\
(\mathrm{GHz})\end{array}$ & Program & $\begin{array}{c}\text { Date } \\
\mathrm{dd} / \mathrm{mm} / \mathrm{yy}\end{array}$ & Conf. & $N_{\text {ant }}$ & $\begin{array}{c}\text { BW } \\
(\mathrm{MHz})\end{array}$ & $\begin{array}{c}\text { TOS } \\
(\mathrm{s})\end{array}$ \\
\hline 1.4 & AH0633 & $11 / 03 / 98$ & $\mathrm{~A}$ & 23 & 100 & 130 \\
4.8 & AG0670 & $09 / 10 / 04$ & $\mathrm{~A}$ & 26 & 100 & 2010 \\
15.9 & AH0633 & $11 / 03 / 98$ & $\mathrm{~A}$ & 27 & 100 & 170 \\
43.3 & AL0618 & $26 / 01 / 04$ & $\mathrm{BC}$ & 26 & 100 & 1320 \\
\hline
\end{tabular}

Table 2. Parameters of images in Fig. 3.

\begin{tabular}{rllrrrrl}
\hline \hline $\begin{array}{r}v_{\text {obs }} \\
(\mathrm{GHz})\end{array}$ & Array & Wt. & $\begin{array}{r}b_{\text {maj }} \\
(\mathrm{mas})\end{array}$ & $\begin{array}{r}b_{\min } \\
(\mathrm{mas})\end{array}$ & $\begin{array}{r}\theta_{\text {pa }} \\
\left({ }^{\circ}\right)\end{array}$ & $\begin{array}{r}S_{\text {peak }} \\
(\mathrm{mJy} / \mathrm{b})\end{array}$ & $\begin{array}{l}\sigma_{\text {rms }} \\
(\mathrm{mJy} / \mathrm{b})\end{array}$ \\
\hline 1.4 & VLA:A & NW & 1580 & 1310 & 7.6 & 862 & 0.3 \\
4.8 & VLA:A & NW & 563 & 404 & 41.7 & 919 & 0.07 \\
15.9 & VLA:A & NW & 156 & 130 & 7.8 & 754 & 0.3 \\
43.3 & VLA:BC & UW & 419 & 145 & 81.2 & 476 & 0.3 \\
\hline 1.6 & VLBA & NW & 10.50 & 4.85 & 1.7 & 710 & 0.07 \\
& VSOP & UW & 6.88 & 1.19 & 31.7 & 536 & 0.3 \\
4.8 & VLBA & NW & 3.93 & 1.81 & -4.5 & 702 & 0.3 \\
& VSOP & NW & 2.79 & 0.99 & 22.3 & 610 & 0.3 \\
& VSOP & UW & 1.81 & 0.17 & 29.1 & 261 & 1.3 \\
15.3 & VLBA & NW & 1.24 & 0.56 & -1.7 & 370 & 0.3 \\
\hline
\end{tabular}

on-source observing time. We also used the 2.3/8.6 GHz visibility data provided by US Naval Observatory (USNO) Radio Reference Frame Image Database (RRFID) ${ }^{2}$. Those observations used 10 VLBA antennas and some additional geodetic antennas. All the VLA data used in this paper were obtained from the NRAO Data Archive ${ }^{3}$. The basic parameters of the VLA observations are summarised in Table 1 . The columns give (1) frequency in $\mathrm{GHz},(2)$ program ID, (3) date as dd/mm/yy, (4) array configuration, (5) antenna numbers, (6) bandwidth in $\mathrm{MHz}$, and (7) total on-source time in seconds. All the VLA observations used 3C 286 as the prime flux density calibrator. After a priori calibrations in AIPS, we performed self-calibration, imaging and model-fitting in Difmap.

\section{Results}

Figure 3 shows all the images of PKS $1402+044$ of the current study. Their parameters are summarised in Table 2. The columns give: (1) frequency in GHz; (2) array and configuration; (3) weighting (NW: natural, UW: uniform), (4-5) size of the synthesised beam in mas; (6) position angle of the major axis in mas; (7) peak brightness in mJy/beam, and (8) image noise level in $\mathrm{mJy} /$ beam $(1 \sigma)$. From the final fringe-fitted VSOP data set we produced two images: (1) an image with all data included (hereafter VSOP image) and (2) an image using only the ground VLBA data at each frequency. In the imaging process, we scaled the gridding weights inversely with the amplitude errors. The VSOP image fidelity is limited, in particular, by the completeness of the $(u, v)$ coverage. In our case, the latter is essentially one-dimensional (see Fig. 1) that leads to a highly elliptical synthesised beam. However, luckily, the highest angular resolution is achieved along the position angle of $\sim-60^{\circ}$, very close to that of the inner pc-scale jet. Thus, the space-ground baselines obtained play an important role in imaging the inner pc-scale jet of PKS $1402+044$

We detect a very weak jet emission extending up to $\sim 150$ mas $(\sim 1 \mathrm{kpc})$ in the high dynamic range $(\sim 10000)$ VLBA image at $1.6 \mathrm{GHz}$ show Fig. 3e. It represents a typical core-jet morphology. We identify a compact core (component A) and five

\footnotetext{
2 http://rorf.usno.navy.mil/RRFID

${ }^{3}$ http://archive.nrao.edu/archive/e2earchive.jsp
}
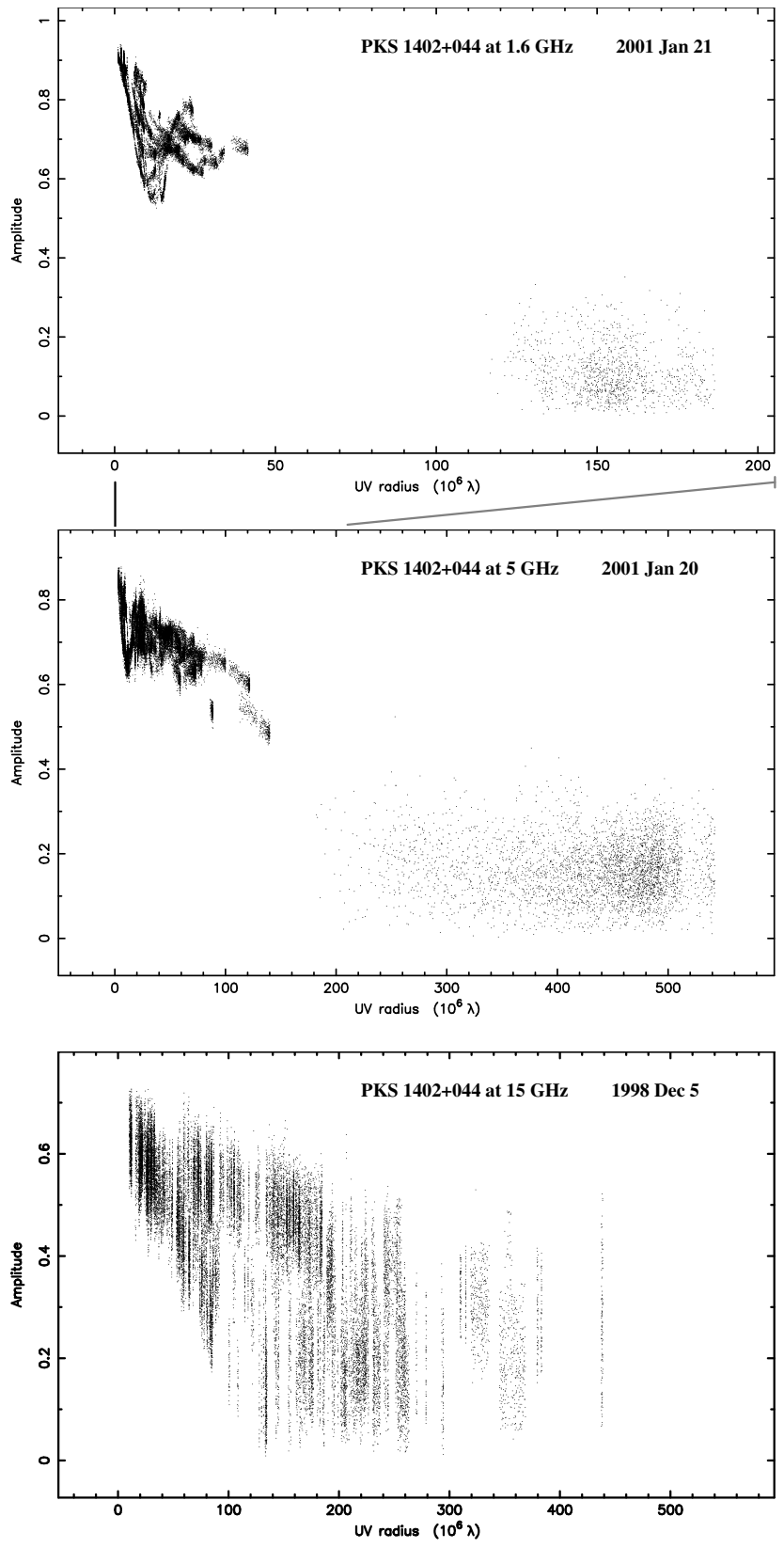

Fig. 2. Correlated flux densities of the source PKS $1402+044$ versus $(u$, v) distance at $1.6 \mathrm{GHz}$ (top VSOP data), $5 \mathrm{GHz}$ (middle VSOP data) and $15 \mathrm{GHz}$ (bottom VLBA data).

jet emission regions (components B - F) using circular Gaussian model-fitting in Difmap. The parameters of the models are listed in Table 3. The columns give: (1) component identification; (2) total flux density of the component; (3-4) radius and position angle of the centre of the component; (5) size of the fitted circular Gaussian model; (6) the smallest detectable size; and (7) brightness temperature in the source frame in $\mathrm{K}$. The error $(1 \sigma)$ is also listed for all the values. The jet shows a wide section between 20 and 70 mas (140-490 pc projected distance). The uniformlyweighted VSOP image shown in Fig. 3f has a higher angular resolution (at the expense of considerably higher image noise) and indicates that components $\mathrm{E}$ and $\mathrm{F}$ are essentially resolved.

The $5 \mathrm{GHz}$ VLBA image in Fig. $3 \mathrm{~g}$ shows a similar structure to the $1.6 \mathrm{GHz}$ VSOP image and the earlier $5 \mathrm{GHz}$ image by Gurvits et al. (1992). The naturally-weighted VSOP image at 

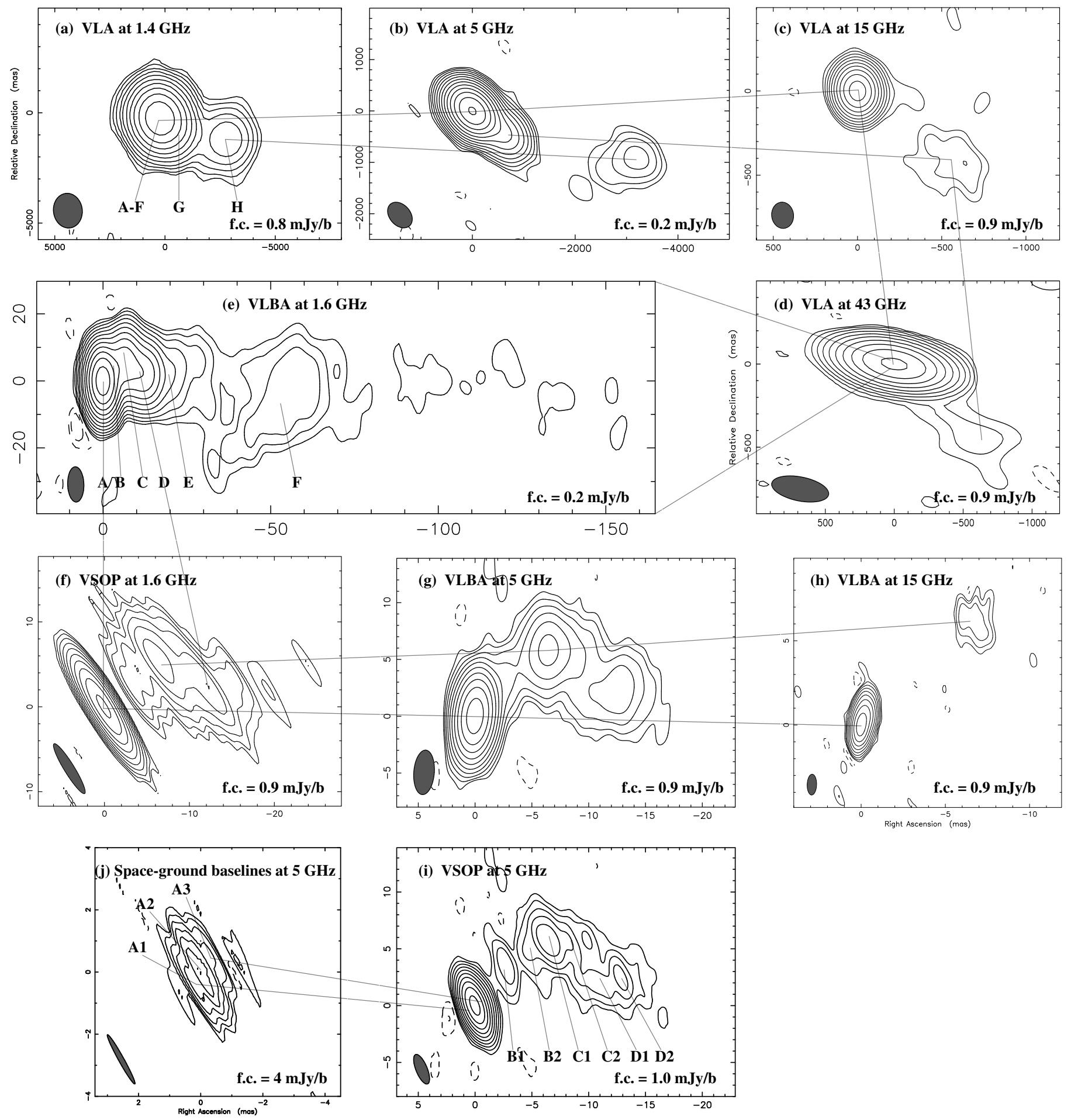

Fig. 3. VLA and VLBI images of PKS 1402+044. The contours are drawn at $-2,-1,1,2, \ldots$, of the respective first contour (f.c.) levels marked in the bottom of each panel. The latter are $\sim 3 \sigma$ of the image thermal noise. The synthesised beams are plotted in the bottom-left corner of each image. The basic parameters of each image are listed in Table 2.

$5 \mathrm{GHz}$ shows that the jet components at $1.6 \mathrm{GHz}$ are resolved into a few subcomponents. Here we have differentiated them with postfix number in the uniformly-weighted space-ground image (Fig. 3j). In this image, the jet appears to be heavily resolved. The core shows a three-component morphology. A weak component marked as A1 appears at the base of the jet and near the brightest component A2. The weakness of component A1 may be due to synchrotron self-absorption considering its high brightness temperature $\left(\sim 10^{12} \mathrm{~K}\right)$. There are two relatively weak jet components, $\mathrm{B} 1$ and $\mathrm{B} 2$, at 1.6 and $5 \mathrm{GHz}$ between the bright components $\mathrm{A}$ and $\mathrm{C}$. At the higher frequency, $15 \mathrm{GHz}$, both components are too weak $(<0.9 \mathrm{mJy} /$ beam $)$ to be detected. Based on the spectrum at frequencies $\leq 5 \mathrm{GHz}$, the extrapolated total flux density of $\mathrm{B} 1+\mathrm{B} 2$ is $\sim 5 \mathrm{mJy}$ at $15 \mathrm{GHz}$. The nondetection of the two components indicates that they have a steeper spectrum $(\alpha<-0.9)$ at frequencies $>5 \mathrm{GHz}$. 
Table 3. The parameters and the brightness temperatures of the fitted Gaussian models.

\begin{tabular}{rrrccrc}
\hline \hline Comp. & $\begin{array}{c}S_{\text {int }} \\
(\mathrm{mJy})\end{array}$ & $\begin{array}{c}r \\
(\mathrm{mas})\end{array}$ & $\begin{array}{c}\theta_{\text {pa }} \\
\left({ }^{\circ}\right)\end{array}$ & $\begin{array}{c}d \\
(\mathrm{mas})\end{array}$ & $\begin{array}{r}d_{\text {lim }} \\
(\mathrm{mas})\end{array}$ & \multicolumn{1}{l}{$T_{\mathrm{b}}$} \\
$(\mathrm{K})$
\end{tabular}

The 1.4 GHz VLA image (Fig. 3a) has the lowest resolution and shows that there is a weak $(\sim 33 \mathrm{mJy})$ component $(\mathrm{H})$ at a distance of $33^{\prime \prime} 22$ and a position angle $-107^{\circ} .4$ from the core, besides the main emission region. It agrees well with earlier MERLIN observations made with the Westerbork Synthesis Radio Telescope (WSRT; Gurvits et al. 1992). The higher sensitivity (0.07 mJy/beam) VLA observations (Fig. 3b) at $5 \mathrm{GHz}$ indicate that component $\mathrm{H}$ has a weak extension toward east. The extension is consistent with the hypothesis that component $\mathrm{H}$ belongs to the jet of PKS $1402+044$. The main emission region can be approximated by component $\mathrm{G}$ and a combination of the inner components $(\mathrm{A}-\mathrm{F})$ in the VLA images. Component $\mathrm{G}$ is also detected at $15 \mathrm{GHz}$ in Fig. 3c and even $43 \mathrm{GHz}$ in Fig. 3d. The highest observing frequency corresponds to the rest-frame emitted frequency of $\sim 180 \mathrm{GHz}$. Arguably, this is one of the rare cases of a profound jet emission at millimetre wavelengths.

\section{Discussion}

\subsection{Spectral properties of the jet}

The resolution $(6.88 \times 1.19$ mas $)$ of the Space VLBI image of PKS $1402+044$ at $1.6 \mathrm{GHz}$ is close $(3.55 \times 1.40$ mas $)$ to that of the ground VLBA image at $5 \mathrm{GHz}$, enabling extraction of spectral index information from a combination of the two images. We restored the 1.6-GHz VSOP image and the 5-GHz VLBA image with a circular Gaussian beam of 4 mas in diameter. The artificial beam increases the beam area by a factor of $\sim 2$ at $1.6 \mathrm{GHz}$ and $\sim 3$ at $5 \mathrm{GHz}$ compared to the areas of the original synthesised beams. Both images were aligned at the strongest component. The shifts in the image centre are less than 0.1 mas ( $\ll 4$ mas resolution). After the alignment, the spectral index was calculated at all pixels with brightness values higher than $1.8 \mathrm{mJy} / \mathrm{beam}$ $(5 \sigma)$ in the $1.6 \mathrm{GHz}$ image and $1.2 \mathrm{mJy} /$ beam $(5 \sigma)$ in the $5 \mathrm{GHz}$ image. A possible core shift between the two frequencies as predicted by Kovalev et al. (2008) does not exceed 1.5 mas. Thus, it does not affect the large-scale spectral distribution.

The final spectral index distribution between $1.6 \mathrm{GHz}$ and $5 \mathrm{GHz}$ is displayed in Fig. 4. It shows a smooth distribution of spectral index on $\sim 20$ mas $(140 \mathrm{pc})$ scale. The spectral index varies from +0.1 in the optically thick base region to -1.0 in the optically thin regions on the western side. To further confirm the variation, we plotted the components spectra in Fig. 5. Here we also used the $2.3 / 8.4 \mathrm{GHz}$ visibility data from the USNO RRFID database. We fitted the VLBI visibility data with three components at each frequency $(1.6,2.3,5,8.4$, and $15 \mathrm{GHz})$. The spectra of the large-scale components $\mathrm{G}$ and $\mathrm{H}$ are also plotted. All spectra can be approximated by a power-law model, $S_{v}=S_{0} v^{\alpha}$. The spectral indices are listed in Table 4 . The spectral steepening 


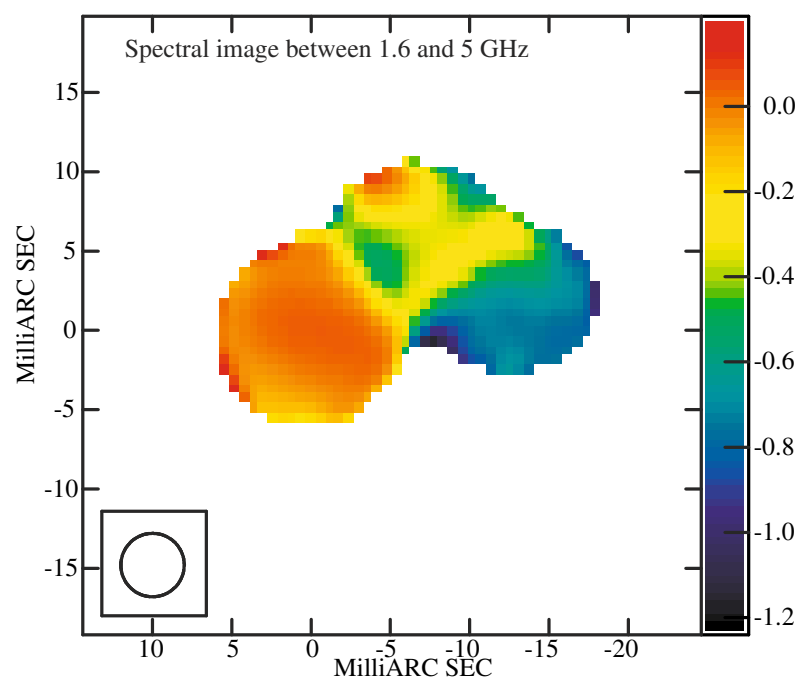

Fig. 4. The spectral index distribution in the jet of the quasar PKS $1402+044$.

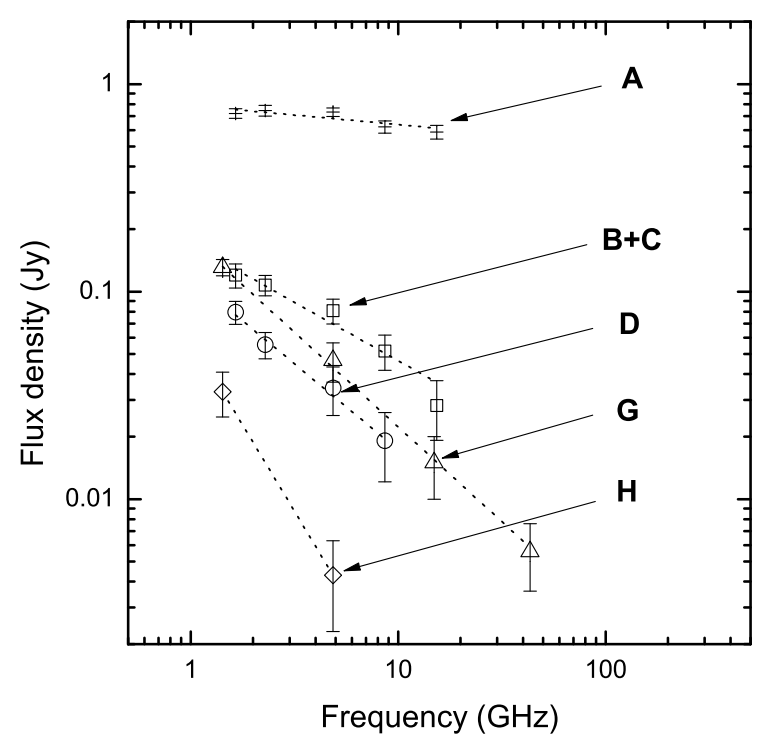

Fig. 5. The component spectra in the jet of the quasar PKS 1402+044. The dotted lines represent the fitted curves with the simple power-law model $S_{v}=S_{0} v^{\alpha}$. The estimated spectral indices are listed in Table 4 .

increases with the increase in the distance from the core. The spectral difference is 0.47 between components $\mathrm{A}$ and $\mathrm{B}+\mathrm{C}$ and reaches 1.57 between component $\mathrm{A}$ and the farthest component $\mathrm{H}$. This spectral index gradient results in the variation in the flux density ratio of the components $(\mathrm{B}+\mathrm{C})$ over $\mathrm{A}$ from $\sim 0.19$ at $1.6 \mathrm{GHz}$ to $\sim 0.05$ at $15 \mathrm{GHz}$. In a sample of sources at various redshifts, an increase in redshift is equivalent to the increase in the intrinsic emitting frequency, $v_{\mathrm{em}}=v_{\mathrm{obs}}(1+z)$. Thus, a decrease in jet-to-core flux density ratio with increase in redshift is expected (Frey et al. 1997). The quasar PKS 1402+044 has a spectral difference of $\sim 0.6$ at the pc scale, which agrees well with the prediction $0.55 \pm 0.43$ by Frey et al. (1997).

\subsection{The mass of the central object of PKS $1402+044$}

The richness of the core-jet morphology in PKS 1402+044 makes it a suitable source for estimating parameters of the
Table 4. Results of the power-law spectral model fits for each component shown in Fig. 5.

\begin{tabular}{llcl}
\hline \hline Comp. & $\begin{array}{l}S_{0} \\
(\mathrm{Jy})\end{array}$ & $\alpha$ & $\chi^{2}$ \\
\hline $\mathrm{A}$ & $0.79 \pm 0.05$ & $-0.09 \pm 0.04$ & 1.19 \\
$\mathrm{~B}+\mathrm{C}$ & $0.17 \pm 0.02$ & $-0.56 \pm 0.10$ & 0.72 \\
$\mathrm{D}$ & $0.12 \pm 0.02$ & $-0.83 \pm 0.19$ & 0.16 \\
$\mathrm{G}$ & $0.18 \pm 0.09$ & $-0.91 \pm 0.09$ & 0.08 \\
$\mathrm{H}$ & $0.06 \pm 0.03$ & $-1.66 \pm 0.40$ & - \\
\hline
\end{tabular}

central black hole. The smallest detectable size for a circular Gaussian component in an image with an rms noise $\sigma_{\mathrm{rms}}$ is defined as (Lobanov 2005):

$d_{\mathrm{lim}}=\frac{2^{2-\beta / 2}}{\pi}\left[\pi b_{\text {maj }} b_{\text {min }} \ln 2 \ln \left(\frac{S_{\mathrm{int}} / \sigma_{\mathrm{rms}}}{S_{\mathrm{int}} / \sigma_{\mathrm{rms}}-1}\right)\right]^{1 / 2}$,

where $S_{\text {int }}$ is the integrated flux density of the component, $b_{\text {maj }}$ and $b_{\min }$ are the major and minor axes of the restoring beam respectively, $\beta=0$ for uniform weighting and $\beta=2$ for naturally weighting. Based on the above criterion, except for the size of the combined component from $\mathrm{A}$ to $\mathrm{F}$ at $43 \mathrm{GHz}$, all the sizes estimated from our VLBI and VLA images in Col. (5) of Table 3 can be taken as the true sizes of the jet emission regions.

If the component size is related to the physical transverse dimension of the jet, the mass of the central object can be estimated assuming that the jet is collimated by the ambient magnetic field of the host galaxy. The jet components A2 and A3 have the best measurements of the width of the jet close to the central object, as they are most likely free of the effect of the adiabatic expanding of the jet and synchrotron self-absorption and have the higher reliability, $d / \sigma_{d}>10$, where $d$ and $\sigma_{d}$ are the angular size and error of the fitted circular Gaussian component. For a jet collimated by the ambient magnetic field $B_{\text {ext }}$ of the host galaxy, the mass of the central object $M_{\mathrm{BH}}$ can be related to the width of the jet $r_{\text {jet }}$ (in pc) according to the following relation (Beskin 1997):

$M_{\mathrm{BH}} \simeq r_{\mathrm{jet}}\left(B_{\mathrm{ext}} / B_{\mathrm{gr}}\right)^{1 / 2} 10^{13} M_{\odot}$,

where $B_{\mathrm{gr}}$ is the magnetic field measured at the Schwarzschild radius $R_{\mathrm{gr}}$ of the central black hole. Equation (2) refers to the transverse dimension of the jet measured at distances comparable to the collimation scale (typically expected to be located at $\left.10^{2}-10^{3} R_{\mathrm{gr}}\right)$. A typical galactic magnetic field is $B_{\mathrm{ext}} \sim 10^{-5} \mathrm{G}$ (Beck 2000) and one can expect to have $B_{\mathrm{gr}} \sim 10^{4} \mathrm{G}$ (Field \& Rogers 1993). Based on these parameters, the mass of the central object is $\sim 10^{9} M_{\odot}$. The main uncertainty of the mass estimation arises from the uncertainty in $B_{\mathrm{gr}}$. However, the dependence of the mass on the value $B_{\mathrm{gr}}$ is rather weak, $M_{\mathrm{BH}} \propto B_{\mathrm{gr}}^{-0.5}$; with the magnetic field varying within 4 orders of magnitude, the estimated central black hole mass varies within two orders of magnitude. The external magnetic field $B_{\text {ext }}$ normally varies within a narrow range around $\left(10^{-5 \pm 1} \mathrm{G}\right)$. Thus, the magnetic field uncertainty should not affect the estimated mass drastically.

\subsection{Brightness temperature}

Based on the parameters of the Gaussian models listed in Table 2, we calculated the brightness temperature of each component using the following formula (Kellermann \& Owen 1988):

$T_{\mathrm{b}}=1.22 \times 10^{12}(1+z) \frac{S_{\mathrm{int}}}{d^{2} v^{2}}$ 


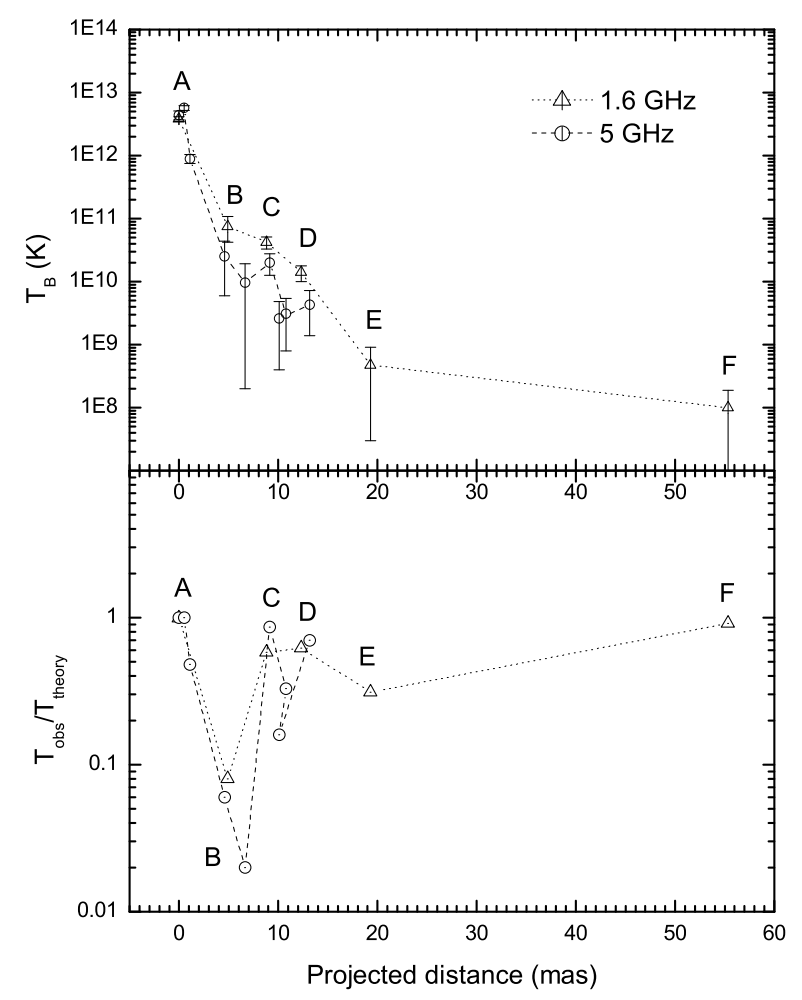

Fig. 6. Brightness temperature variations along the jet in PKS 1402+044 (top) and comparison with the model predicted value (bottom, Marscher 1990). The component identification is described in Fig. 3. The triangles represent the estimated brightness temperature at $1.6 \mathrm{GHz}$; the circles represent the brightness temperature at $5 \mathrm{GHz}$.

where $S_{\text {int }}$ is the integrated flux density in Jy, $d$ the size of a circular Gaussian component in mas, and $v$ the observing frequency in GHz. The estimated brightness temperatures are listed in the last column of Table 2. Among these components, the component $\mathrm{A} 2$ has the highest brightness temperature, $T_{\mathrm{B}}=$ $(5.7 \pm 0.5) \times 10^{12} \mathrm{~K}$, which is somewhat higher than the inverse Compton limit $\left(\sim 10^{12} \mathrm{~K}\right.$; Kellermann \& Pauliny-Toth 1969) but still 10-times lower than the currently known highest value of $5.8 \times 10^{13} \mathrm{~K}$ found in the BL Lac object AO $0235+164$ by Frey et al. (2000). In the equipartition jet model of Blandford \& Königl (1979), the limiting brightness temperature is about $3 \times 10^{11} \delta^{5 / 6} \mathrm{~K}$, where $\delta$ is the Doppler factor. Comparing the theoretical value with the estimated brightness temperature of the component A2, we can obtain a conservative lower limit to the Doppler factor of the inner jet, $\delta \approx 23.7$.

The variation in the observed brightness temperature with increasing distance from the core is plotted in Fig. 4. Following Marscher (1990), we assume that each of the jet components is an independent plane shock in which the radio emission is dominated by adiabatic energy losses. The jet plasma has a powerlaw energy distribution, $N(E) \mathrm{d} E \propto E^{-s} \mathrm{~d} E$. The magnetic field varies as $B \propto l^{-a}$, where $l$ is the distance from the central object. The Doppler factor is assumed to vary weakly throughout the jet. Under these assumptions, one can relate the brightness temperature $T_{\mathrm{b}, \text { jet }}$ of each jet component to the brightness temperature of the core $T_{\mathrm{b} \text {,core }}$ :

$T_{\mathrm{b}, \text { jet }}=T_{\mathrm{b}, \text { core }}\left(d_{\text {jet }} / d_{\text {core }}\right)^{-\epsilon}$,

where $d$ represents the measured size of the core and jet features and $\epsilon=[2(2 s+1)+3 a(s+1)] / 6$ (Lobanov et al. 2001). We take $s=2.06$ corresponding to the synchrotron emission with the spectral index of component $\mathrm{B}+\mathrm{C} \alpha=0.53$, and $a=1$ corresponding to the transverse orientation of magnetic field in the jet (Lobanov et al. 2001). At each frequency, we take the brightest component as the core. Comparing with the measured one, we plotted the ratio of $T_{\text {obs }} / T_{\text {theory }}$ versus the distance. The largest discrepancies occur with components $\mathrm{B}$ at $1.6 \mathrm{GHz}, \mathrm{B} 1$, and B2 at $5 \mathrm{GHz}$. These components have ratios $d / \sigma_{d}=5$ at $1.6 \mathrm{GHz}$ and $d / \sigma_{d} \leq 3$ at $5 \mathrm{GHz}$. The ratios indicate that the discrepancy may be caused by uncertainties in the size estimates for strongly resolved components or inhomogeneities in the plasma over such large emitting regions.

\subsection{Proper motion}

Using an earlier VLBI observation at $5 \mathrm{GHz}$ in 1986 by Gurvits et al. (1992), we tried to estimate the proper motion in PKS $1402+044$ over the time interval of 15 years. We also fitted the $(u, v)$ visibility data with 6 Gaussian models and found that the position offset is within the one fifth of the beam $(10 \times 2$ mas in $\mathrm{PA}-2^{\circ}$ ) of the image in 1986 in the east-west direction where the image has the higher resolution, and there is no consistent shift. Thus, an upper limit of the apparent proper motion $\mu$ in the EW direction is 0.03 mas $\mathrm{yr}^{-1}$. This corresponds to the apparent velocity upper limit of $\beta_{\text {app }}=3 c$ based on the relation $\beta_{\text {app }}=1.58 \times 10^{-2} \mu D_{\mathrm{A}}(1+z)$ (Kellermann et al. 2004), where the angular size distance $D_{\mathrm{A}}$ is measured in Mpc, $\mu$ in mas $\mathrm{yr}^{-1}$ and $\beta_{\text {app }}$ in the unit of the speed of light, $c$.

Using the determined lower limit $\delta=23.7\left(\delta \gg \sqrt{\beta_{\text {app }}^{2}+1}\right)$ and the following equations (e.g. Hong et al. 2008):

$$
\begin{gathered}
\gamma=\frac{\beta_{\text {app }}^{2}+\delta^{2}+1}{2 \delta}, \\
\tan \phi=\frac{2 \beta_{\text {app }}}{\beta_{\text {app }}^{2}+\delta^{2}-1},
\end{gathered}
$$

a lower limit to the Lorentz factor $\gamma \approx 12$ of the jet and an upper limit to the viewing angle to the line of sight $\phi \approx 1^{\circ}$ can be determined. All the estimates suggest that PKS $1402+044$ is a relativistically beamed radio sources.

\section{Summary}

Based on multi-frequency VLBI (1.6, 2.3, 5, 8.4, and $15 \mathrm{GHz})$ observations including dual-frequency (1.6 and $5 \mathrm{GHz}$ ) VSOP observations and VLA $(1.4,5,15$, and $43 \mathrm{GHz})$ observations of the high-redshift quasar PKS $1402+044$, we draw the following conclusions.

1. The quasar PKS 1402+044 demonstrates a well-defined core-jet morphology that can be traced out to $\sim 23 \mathrm{kpc}$ from the source core.

2. The radio spectral index distribution and the component spectra prove that the jet has the steeper spectrum with increasing distance from the core.

3. Based on the measurement of the transverse size of the jet, and assuming that the external magnetic field collimates the jet model, the mass of the central object is estimated as $\sim 10^{9} M_{\odot}$.

4. PKS $1402+044$ has a bright core $\left(5.7 \times 10^{12} \mathrm{~K}\right)$, and the observed brightness temperature variation is basically consistent with the shock-in-jet model. 
5. No firm detection of a proper motion in the jet can be made. An upper limit of the apparent proper motion in the eastwest direction is 0.03 mas $\mathrm{yr}^{-1}$, corresponding to the apparent speed of $3 c$.

6. Based on the lower limit of the Doppler factor $\delta=23.7(\delta \gg$ $\left.\sqrt{\beta_{\text {app }}^{2}+1}\right)$, we estimate the lower limit to the Lorentz factor $\gamma=12$ and the upper limit to the viewing angle of the inner jet to the line of sight as $\phi=1^{\circ}$.

Acknowledgements. We are grateful to Alan Fey for providing us with the S/Xband USNO RRFID data, Richard Schilizzi and Ken Kellermann for their assistance at various stages of the project, PI's and the teams of VLA observations used in this work. The original idea for this project was conceived in discussions with Ivan Pauliny-Toth. This research was partly supported by the Natural Science Foundation of China (NSFC10473018 and NSFC10333020). Jun Yang and Xiaoyu Hong are grateful to the KNAW - CAS grant 07DP010. Sándor Frey acknowledges the OTKA K72515 and HSO TP314 grants. We gratefully acknowledge the VSOP Project, which was led by the Institute of Space and Astronautical Science (Japan) in cooperation with many agencies, institutes, and observatories around the world. The National Radio Astronomy Observatory is a facility of the National Science Foundation operated under cooperative agreement by Associated Universities, Inc. This research has made use of NASA's Astrophysics Data System, NASA/IPAC Extragalactic Database (NED), and the United States Naval Observatory (USNO) Radio Reference Frame Image Database (RRFID).

\section{References}

Beck, R. 2000, in Perspectives on Radio Astronomy: Science with Large Antenna Arrays, ed. M. P. van Haarlem (Dwingeloo: ASTRON), 249
Beskin, V. S. 1997, Physi.-Uspekhi, 40, 659

Blandford, R. D., \& Königl, A. 1979, ApJ, 232, 34

Cotton, W. D. 1995, in Very Long Baseline Interferometry and the VLBA, ed.

J. A. Zensus, P. J. Diamond, \& P. J. Napier, ASP Conf. Ser., 82, 189

Frey, S., Gurvits, L. I., Kellermann, K. I., Schilizzi, R. T., \& Pauliny-Toth, I. I. K. 1997, A\&A, 325, 511

Frey, S., Gurvits, L. I., Altschuler, D. R. et al. 2000, PASJ, 52, 975

Field, G. B., \& Rogers, R. D. 1993, ApJ, 403, 94

Hirabayashi, H., Hirosawa, H., Kobayashi, H., et al. 1998, Science, 281, 1825

Gurvits, L. I., Kardashev, N. S., Popov, M. V., et al. 1992, A\&A, 260, 82

Gurvits, L. I., Kellermann, K. I., \& Frey, S. 1999, A\&A, 342, 378

Hong, X.-Y., Sun, C. -H., Zhao, J.-H., et al. 2008, Chinese J. Astron. Astrophys., in press

Kellermann, K. I., \& Pauliny-Toth, I. I. K. 1969, ApJ, 155, L71

Kellermann, K. I., \& Owen, F. N. 1988, in Galactic and Extragalactic Radio Astronomy, ed. G. L. Verschuur, \& K. I. Kellermann (Springer: Berlin), 563

Kellermann, K. I., Vermeulen, R. C., Zensus, J. A., Cohen, M. H., \& West, A. 1999, New A Rev., 43, 757

Kellermann, K. I., Lister, M. L., Homan, D. C., et al. 2004, ApJ, 609, 539

Kovalev, Y. Y., Lobanov, A. P., Pushkarev, A. B., \& Zensus, J. A. 2008, A\&A, 483,759

Lobanov, A. P., Gurvits, L. I., Frey, S., et al. 2001, ApJ, 547, 714

Lobanov, A. P., Krichbaum, T. P., Witzel, A., \& Zensus, J. A. 2006, PASJ, 58, 253

Lobanov, A. P. 2005, [arXiv: astro-ph/0503225]

Marscher, A. P. 1990, in Parsec-Scale Radio Jets, ed. J. A. Zensus, \& T. J. Pearson (Cambridge: Cambridge Univ. Press), 236

Paragi, Z., Frey, S., Gurvits, L. I., et al. 1999, A\&A, 344, 51

Riess, A. G., Strolger, L.-G., Tonry, J., et al. 2004, ApJ, 607, 665

Siebert, J., Brinkmann, W., Drinkwater, M. J., et al. 1998, MNRAS, 301, 261

Shepherd, M. C., Pearson, T. J., \& Taylor, G. B. 1994, BAAS, 26, 987

Thompson, R. J., Shelton, R. G., \& Arning, C. A. 1998, AJ, 115, 2587 